\title{
The usefulness of end-tidal carbon dioxide monitoring during apnea test in brain-dead patients
}

\author{
Ha Yeon Kim ${ }^{1}$, Gaab-Soo Kim ${ }^{1}$, Young Hee Shin ${ }^{1}$, and So Ra Cha ${ }^{2}$ \\ ${ }^{1}$ Department of Anesthesiology and Pain Medicine, ${ }^{2}$ Organ Transplantation Center, Samsung Medical Center, Sungkyunkwan \\ University School of Medicine, Seoul, Korea
}

Background: The apnea test (AT) is essential to confirming the diagnosis of brain death, but critical complications can occur if the AT is maintained over a long period. To minimize the AT period, we used end-tidal carbon dioxide $\left(\mathrm{ETCO}_{2}\right)$ monitoring because $\mathrm{ETCO}_{2}$ is closely correlated with partial pressure of arterial carbon dioxide $\left(\mathrm{PaCO}_{2}\right)$. The aim of the present study is to evaluate the usefulness of $\mathrm{ETCO}_{2}$ monitoring during apnea testing.

Methods: We reviewed 61 patients who were pronounced brain dead at our hospital from July 2009 to December 2012. The subjects were divided into two groups: the N-group, in which capnography was not used, and the C-group, in which capnography was used to monitor $\mathrm{ETCO}_{2}$. In the $\mathrm{C}$-group, whenever arterial blood was sampled, the $\mathrm{PaCO}_{2}-\mathrm{ETCO}_{2}$ gradients were calculated and the ventilator setting adjusted to maintain normocapnia prior to apnea testing.

Results: Twenty-eight subjects in the N-group and twenty-nine subjects in the C-group were included. The gender ratio, age, and cause of brain death were not different between the two groups. Prior to the AT, the normocapnia ratio was higher in the C-group than in the N-group. During the AT, the total test period was shorter in the C-group. Moreover, systolic blood pressure increased in the $\mathrm{C}$-group and decreased in the $\mathrm{N}$-group during apnea testing.

Conclusions: $\mathrm{ETCO}_{2}$ monitoring during AT allows the $\mathrm{PaCO}_{2}$ level to be predicted, which reduces the duration of the test and stabilizes systolic blood pressure. Thus, with $\mathrm{ETCO}_{2}$ monitoring, the AT can be fast and safe. (Korean J Anesthesiol 2014; 67: 186-192)

Key Words: Apnea, Blood gas analysis, Brain death, Capnography, Carbon dioxide.

Received: December 12, 2013. Revised: 1st, January 26, 2014; 2nd, April 6, 2014; 3rd, April 17, 2014. Accepted: April 19, 2014.

Corresponding author: Gaab-Soo Kim, M.D., Ph.D., Department of Anesthesiology and Pain Medicine, Samsung Medical Center, Sungkyunkwan University School of Medicine, 81, Irwon-ro, Gangnam-gu, Seoul 135-710, Korea. Tel: 82-2-3410-0360, Fax: 82-2-3410-0361, E-mail: gskim@ skku.edu

(c) This is an open-access article distributed under the terms of the Creative Commons Attribution Non-Commercial License (http:// creativecommons.org/licenses/by-nc/3.0/), which permits unrestricted non-commercial use, distribution, and reproduction in any medium, provided the original work is properly cited. 


\section{Introduction}

Brain death is the irreversible loss of all brain functions, including the brain stem, caused by organic brain lesions $[1,2]$. Although the clinical criteria of brain death differ somewhat across countries, the apnea test is a mandatory step in many countries, including the U.S., the U.K., and Japan. The apnea test is undertaken to demonstrate the absence of spontaneous breathing when the partial pressure of arterial carbon dioxide $\left(\mathrm{PaCO}_{2}\right)$ reaches a target level for sufficient stimulation of the respiratory center. However, complications such as respiratory acidosis, hypotension, hypoxia, and cardiac arrhythmia may occur if the confirmation of target $\mathrm{PaCO}_{2}$ takes a long time [3-5]. These complications can cause insufficient organ perfusion and increase the failure rate of organ procurement [6,7]. Therefore, it is important to minimize the performance time of apnea testing.

The American Academy of Neurology (AAN) established prerequisites to reduce the worsening of donor condition during the apnea test. One prerequisite is to maintain normocapnia ( $\mathrm{PaCO}_{2} 35-45 \mathrm{mmHg}$ ) prior to apnea testing. This is intended to prevent respiratory acidosis or alkalosis and minimize complications due to abnormal $\mathrm{PaCO}_{2}$. To maintain normocapnia and confirm the target level of $\mathrm{PaCO}_{2}$, serial arterial blood gas analysis (ABGA) is necessary.

We planned to use end-tidal carbon dioxide $\left(\mathrm{ETCO}_{2}\right)$ monitoring to decrease the frequency of $\mathrm{ABGA}$ and the performance time of apnea testing because $\mathrm{ETCO}_{2}$ is closely correlated with $\mathrm{PaCO}_{2}$. With continuous monitoring of $\mathrm{ETCO}_{2}$ by capnography, we are able to predict $\mathrm{PaCO}_{2}$ indirectly [8-11] and control $\mathrm{PaCO}_{2}$ without repeated ABGA. Based on these assumptions, the intensive care unit team in our hospital furnished capnography and has been using capnography for deceased donors since November 2011. Therefore, in this study, we investigated the usefulness of capnography during the apnea test. The aim of the present study is to evaluate whether $\mathrm{ETCO}_{2}$ monitoring reduces the performance time and complications during the apnea test.

\section{Materials and Methods}

After obtaining the approval of the Institutional Review Board of our hospital, we investigated patients who were declared brain dead for organ donation from July 2009 to December 2012. We collected computerized and manual medical records of 61 patients from 14 to 75 years old.

After arrival in the intensive care unit (ICU), all subjects were managed under the protocol of the ICU, and vital signs (blood pressure, pulse rate, and oxygen saturation) were recorded. Blood pressure was measured at the radial artery or femoral artery, and samples for ABGA were obtained through this ar- tery. After mainstream capnography (CAPNOSTAT M2501A ${ }^{\circledR}$, Philips, Boblingen, Germany) was available, differences between $\mathrm{PaCO}_{2}$ and $\mathrm{ETCO}_{2}$ were calculated, and the ventilator setting was adjusted to maintain the calculated $\mathrm{PaCO}_{2}$ within the normal range prior to the apnea test. Blood pressure was controlled with dopamine, norepinephrine, and vasopressin, and the target systolic blood pressure was more than $100 \mathrm{mmHg}$.

Neurologic examination for the declaration of brain death was performed twice, and the apnea test was performed at the end of each examination. In accordance with the apnea test protocol, subjects were preoxygenated with $100 \%$ oxygen for 10 minutes prior to the test. The beginning of the test was defined as the time of disconnecting subjects from the ventilator, and $100 \%$ oxygen was delivered to the endotracheal tube of subjects via the T-piece. The physician looked closely for respiratory movement, and $\mathrm{ETCO}_{2}$ was monitored if capnography was used.

ABGA was performed 1-3 minutes after the beginning of the apnea test. If $\mathrm{PaCO}_{2}$ was not greater than $50 \mathrm{mmHg}$, additional ABGA was performed at intervals of 1-3 minutes. When $\mathrm{PaCO}_{2}$ reached the target value and there was no spontaneous respiratory movement, the apnea test was ended and declared positive. If serious complications, such as severe hypoxemia (pulse oximetry saturation $\left[\mathrm{SpO}_{2}\right]<90 \%$ ), hypotension (systolic blood pressure $[\mathrm{SBP}]<90 \mathrm{mmHg}$ ), or critical cardiac arrhythmia, occurred before or during the apnea test, the test was terminated. If $\mathrm{PaCO}_{2}$ did not reach the target level up to that time, a test for cerebral blood flow (transcranial Doppler ultrasonography or cerebral angiography) was used to replace the apnea test.

The subjects were divided into two groups: the N-group, in which capnography was not used, and the C-group, in which capnography was used to monitor $\mathrm{ETCO}_{2}$. The time to target $\mathrm{PaCO}_{2}$ (min) was defined as the period from the beginning of apnea test to the first ABGA sampling of the target $\mathrm{PaCO}_{2}$. The total apnea test period ( $\mathrm{min}$ ) was defined as the period from the beginning of the test to the actual end of the test after confirming the target $\mathrm{PaCO}_{2}$. The attending ICU nurse recorded the arterial blood pressure, pulse rate, and pulse oximetry saturation at the beginning and end of the apnea test in all subjects.

SPSS for Windows (version 18.0, SPSS Inc., Chicago, IL, USA) was used for statistical analysis. Categorical variables were analyzed using the chi-square test or Fisher's exact test. Continuous variables were tested for normality using the KolmogorovSmirnov test. Then, the variables showing normality were analyzed using the Student's t-test and expressed as the mean \pm standard deviation. The variables not showing normality were analyzed using the Mann-Whitney test and expressed as the median (25th percentile-75th percentile). All $\mathrm{P}$ values were calculated using a 2-tailed test, and a P value of less than 0.05 was considered statistically significant. 


\section{Results}

The N-group consisted of 31 patients, and the C-group had 30 patients. Three $(9.7 \%)$ patients in the $\mathrm{N}$-group were excluded due to uncorrected hypotension ( $\mathrm{SBP}<90 \mathrm{mmHg}$ ) before the apnea test, and one patient $(3.3 \%)$ in the C-group was excluded due to hypoxemia $\left(\mathrm{SpO}_{2}<90 \%\right)$ before the apnea test. Therefore, the final sample comprised 28 patients in the N-group and 29 patients in the C-group. No differences were observed between the two groups in gender, age, and cause of brain death (Table 1).

Because the apnea test was performed twice in each patient, ABGA results and hemodynamic variables are arranged separately for each test (Table 2). The ratio of normal $\mathrm{PaCO}_{2}$ before the test (\%), total test time ( $\mathrm{min})$, and time to target $\mathrm{PaCO}_{2}$ (min) were significantly different between the two groups. The SBP of the $\mathrm{N}$-group decreased during the test $\left(1^{\text {st }}\right.$ AT: $-9.0 \pm 26.2 \mathrm{mmHg}$, $2^{\text {nd }}$ AT: $-12.4 \pm 26.8 \mathrm{mmHg}$ ), while the SBP of the C-group in-

Table 1. Patient Demographics and Etiology of Brain Death

\begin{tabular}{lccc}
\hline & $\begin{array}{c}\text { N-group } \\
(\mathrm{n}=28)\end{array}$ & $\begin{array}{c}\text { C-group } \\
(\mathrm{n}=29)\end{array}$ & P value \\
\hline Gender $(\mathrm{M} / \mathrm{F})$ & $18 / 10$ & $21 / 8$ & 0.509 \\
Age $(\mathrm{yr})$ & $46.3 \pm 15.8$ & $46.4 \pm 15.0$ & 0.975 \\
Cause of Brain death & 10 & 7 & 0.248 \\
Hypoxic encephalopathy & 9 & 12 & 0.585 \\
Subarachnoid hemorrhage & 8 & 8 & 0.535 \\
Intracerebral hemorrhage & 0 & 1 & 1.000 \\
Ischemic stroke & 1 & 1 & 0.746 \\
Subdural hemorrhage & & & \\
\hline
\end{tabular}

Data are expressed as number and mean \pm SD. N-group: patients in which capnography was not used, C-group: patients in which capnography was used to monitor end tidal carbon dioxide. No significant difference was observed between the two groups. creased during the test $\left(1^{\text {st }}\right.$ AT: $9.1 \pm 27.7 \mathrm{mmHg}, 2^{\text {nd }}$ AT: $5.3 \pm 31.2$ $\mathrm{mmHg}$ ). The SBP changes during the apnea test were significantly different between the two groups ( $1^{\text {st }}$ AT P value: 0.014 , $2^{\text {nd }}$ AT P value: 0.026$)$. During the test, there were fewer subjects with SBP less than $100 \mathrm{mmHg}$ in the C-group than in the $\mathrm{N}$ group, although there were no significant differences. During the test, there were fewer subjects with $\mathrm{SpO}_{2}$ less than $90 \%$ in the C-group than in the $\mathrm{N}$-group, and $\mathrm{PaO}_{2}$ was less decreased in the C-group than in the $\mathrm{N}$-group, but there was no statistical significance.

\section{Discussion}

At present, about 10 countries approve a legal definition of brain death; these include the U.S., Canada, France, Japan, and Taiwan. Furthermore, more than 30 countries allow organ transplantation from brain dead patients. In 1968, Harvard Medical School set forth criteria for diagnosis brain death, and since then, various countries have furnished their own guidelines for the diagnosis of brain death. Prerequisites (i.e., types of confirmatory and mandatory tests for the declaration of brain death) differ slightly, but the differences are minimal from a medical standpoint. In 1993, the Korean Medical Association established criteria for organ transplantation, and in February 1999, The Act on Organ Transplantation was enacted [12]. In fact, however, organ transplantation had been performed since 1992, before the legislation passed; in spite of the lack of legal recognition of brain death, medical professionals approved and performed organ transplantation. The number of organ transplantations from brain dead patients has increased steadily, and during 2012, there were 409 brain dead donors [13].

The legislated rules for the diagnosis of brain death proposed

Table 2. Blood Gas and Cardiovascular Parameters prior to and during the Apnea Test

\begin{tabular}{|c|c|c|c|c|c|c|}
\hline & \multicolumn{2}{|c|}{ First time } & \multirow[b]{2}{*}{$P$ value } & \multicolumn{2}{|c|}{ Second time } & \multirow[b]{2}{*}{$P$ value } \\
\hline & $\begin{array}{l}\text { N-group } \\
(\mathrm{n}=28)\end{array}$ & $\begin{array}{l}\text { C-group } \\
(\mathrm{n}=29)\end{array}$ & & $\begin{array}{l}\text { N-group } \\
(\mathrm{n}=28)\end{array}$ & $\begin{array}{l}\text { C-group } \\
(\mathrm{n}=29)\end{array}$ & \\
\hline \multicolumn{7}{|l|}{ Prior to AT } \\
\hline Normocapnia (n) & 15 & 29 & $<0.001$ & 19 & 29 & 0.001 \\
\hline $\mathrm{SBP}(\mathrm{mmHg})$ & $132.8 \pm 18.5$ & $133.3 \pm 27.0$ & NS & $128.4 \pm 17.3$ & $132.0 \pm 22.7$ & NS \\
\hline $\mathrm{PaO}_{2}(\mathrm{mmHg})$ & $259.8(144.5-439.3)$ & $268.3(132.2-455.7)$ & NS & $217.2(120.9-392.2)$ & $313.8(155.9-489.7)$ & NS \\
\hline \multicolumn{7}{|l|}{ During AT } \\
\hline SBP < $100(\mathrm{mmHg})$ & $6 / 28$ & $4 / 29$ & NS & $7 / 28$ & $2 / 29$ & NS \\
\hline $\mathrm{SpO}_{2}<90 \%(\mathrm{n})$ & $3 / 28$ & $1 / 29$ & NS & $4 / 28$ & $4 / 29$ & NS \\
\hline Total AT period (min) & $5(4-6)$ & $3(1-3)$ & $<0.001$ & $4(3.5-5)$ & $3(1-3)$ & $<0.001$ \\
\hline Time to target $\mathrm{CO}_{2}(\mathrm{~min})$ & $2(1-4)$ & $1(1-1)$ & $<0.001$ & $2(1.25-4)$ & $2(2-2)$ & $<0.001$ \\
\hline$\Delta \mathrm{SBP}(\mathrm{mmHg})$ & $-9.0 \pm 26.2$ & $9.1 \pm 27.7$ & 0.014 & $-12.4 \pm 26.8$ & $5.3 \pm 31.2$ & 0.026 \\
\hline$\Delta \mathrm{PaO}_{2}(\mathrm{mmHg})$ & $-50.9 \pm 91.4$ & $-8.7 \pm 94.5$ & NS & $-35.2 \pm 104.1$ & $-14.4 \pm 81.2$ & NS \\
\hline
\end{tabular}

Data are expressed as number and mean $\pm \mathrm{SD}$ or median (interquartile range). NS: not significant $(\mathrm{P}>0.05), \mathrm{AT}$ : apnea test, $\mathrm{PaO} \mathrm{O}_{2}$ : arterial oxygen partial pressure, $\mathrm{SpO}_{2}$ : pulse oximetry saturation, Total AT period: time from beginning to end of apnea test. Time to target $\mathrm{CO}_{2}$ : time from beginning of apnea test to first ABGA sampling proved $\mathrm{PaCO}_{2}$ greater than $50 \mathrm{mmHg}$. $\triangle \mathrm{SBP}$ : systolic blood pressure when ending of apnea test - systolic blood pressure when beginning of apnea test. $\Delta \mathrm{PaO}_{2}: \mathrm{PaO}_{2}$ at end of apnea test $-\mathrm{PaO}_{2}$ when beginning apnea test. 
by the Korea Medical Association list seven categories. In these rules, the apnea test for evaluating the recovery of respiratory drive is described as follows.

"After preoxygenation with 100\% oxygen or 95\% oxygen and $5 \%$ carbon dioxide for 10 minutes, disconnect the patient from the ventilator and deliver $6 \mathrm{~L} / \mathrm{min}$ of $100 \%$ oxygen through the endotracheal tube. Look closely for 10 minutes, and if respiratory movements are absent in spite of $\mathrm{PaO}_{2}>50 \mathrm{mmHg}$, it is determined that spontaneous respiration cannot be revived. If the apnea test was not performed completely or was stopped midway, accessorial tests such as a cerebral blood flow study should be performed."

Although the apnea test is a confirmatory test in various countries, the target $\mathrm{PaCO}_{2}$ level during the test differs between countries. While some countries, such as the U.S., Canada, and Germany, set the target $\mathrm{PaCO}_{2}$ level at $60 \mathrm{mmHg}$, other countries such as Korea, the U.K., Switzerland, and Portugal set $50 \mathrm{mmHg}$ as the target $\mathrm{PaCO}_{2}$ level. These differences are not meaningful from a medical standpoint, given that the optimal level for sufficiently stimulating the respiratory center is unknown [14]. However, based on the difference in target $\mathrm{PaCO}_{2}$, we expect to be able to reduce the duration of the apnea test more effectively in Korea than in other countries.

The apnea test can cause serious complications such as severe respiratory acidosis, hypotension, and hypoxemia. As these can worsen the donor's condition and increase the failure rate of organ procurement, we should optimize the donor's condition and reduce the period of apnea testing. However, because the rules enacted by law do not specify patient condition in detail, it is unfeasible to perform the apnea test based only on these rules. Each hospital therefore sets detailed guidelines for the declaration of brain death. In many hospitals, such guidelines are based on the AAN guidelines [15]. Our hospital also set guidelines based on those of the AAN (Table 3). Our guidelines recommend maintaining a normal $\mathrm{PaCO}_{2}$ level $(35-45 \mathrm{mmHg})$ before the apnea test and preventing severe hypotension and hypoxemia. If this is difficult, the test should be terminated and replaced with an alternative test. However, to maintain $\mathrm{PaCO}_{2}$ within a normal range, repeated ABGA samplings are needed, which causes increased labor and cost. We devised capnography to replace ABGA samplings to check $\mathrm{PaCO}_{2}$ levels. Up to now, we could not find any study on the usefulness of capnography in brain dead donors. Thus, we planned a study on the usefulness of capnography in apnea testing.

The human body releases carbon dioxide $\left(\mathrm{CO}_{2}\right)$ accumulated in the process of metabolism by ventilation through the airway. Therefore, $\mathrm{PaCO}_{2}$ can be predicted by the measurement of $\mathrm{ETCO}_{2}$ using capnography. In normal lungs, $\mathrm{PaCO}_{2}$ is higher than $\mathrm{ETCO}_{2}$ by 2 to $5 \mathrm{mmHg}$. This is why dead space in lungs dilutes the $\mathrm{CO}_{2}$ concentration in the actually ventilated amount of $\mathrm{CO}_{2}$. Dead space can be divided into anatomic dead space and physiologic dead space. Anatomic dead space is not part of the ventilating airways, such as terminal bronchioles and alveoli, but rather a part of the conducting airways. Dead space can also exist in the distal part of anatomic dead space in the presence of ventilation-perfusion mismatch; this is called physiologic dead space. Because anatomic dead space is fixed whereas physiologic dead space changes with variation in ventilation and perfusion, if physiologic dead space increases, the gradient of $\mathrm{PaCO}_{2}$ and $\mathrm{ETCO}_{2}$ increases [16-18]. In research involving patients on longterm mechanical ventilation, such as brain dead donors, the gradients increased in those patients more than in patients with normal lung condition [19-21]. In studies on brain dead donors, Vivien et al. [18] reported $9 \pm 4 \mathrm{mmHg}$ and Sharpe et al. [22] reported $7 \pm 4 \mathrm{mmHg}$. From these results, it can be seen that brain dead donors have a larger gradient of $\mathrm{PaCO}_{2}$ and $\mathrm{ETCO}_{2}$ than do patients with normal lung condition. In the present study, the gradient of $\mathrm{PaCO}_{2}$ and $\mathrm{ETCO}_{2}$ is also large: $7 \pm 5 \mathrm{mmHg}$ the first time and $6 \pm 6 \mathrm{mmHg}$ the second time. Such results are explained by the fact that long-term mechanical ventilation increases atelectasis and ventilation-perfusion mismatch [23].

In the C-group, we calculated the gradient of $\mathrm{PaCO}_{2}$ and $\mathrm{ETCO}_{2}$ whenever ABGA was sampled, while continuously monitoring $\mathrm{ETCO}_{2}$ in each patient before the apnea test. Moreover, by taking the gradient into consideration, we adjusted mechanical ventilation to maintain $\mathrm{PaCO}_{2}$ within the normal range. Thus, we were able to monitor $\mathrm{PaCO}_{2}$ continuously in the C-group in contrast to the $\mathrm{N}$-group, in which $\mathrm{PaCO}_{2}$ was monitored intermittently by ABGA samplings. As a result, the ratio of normal $\mathrm{PaCO}_{2}$ was $100 \%$ in the C-group both times, but in the

Table 3. Guidelines for the Apnea Test

(1) Preoxygenate with $100 \%$ oxygen for 10 minutes while keeping normocapnia

(2) Obtain a baseline ABGA

(3) Disconnect the patient from the ventilator and deliver $100 \% \mathrm{O}_{2}$ through the endotracheal tube. Look closely for respiratory movements for this period.

(4) Repeat blood gas sampling for a period of time (1-5 minutes) within 10 minutes according to ABGA result.

(5) If respiratory movements are absent in spite of $\mathrm{PaO}_{2}>50 \mathrm{mmHg}$, the apnea test result is positive.

(6) If arterial hypotension (systolic blood pressure $<90 \mathrm{mmHg}$ ) or $\mathrm{SpO}_{2}<90 \%$ is present and cannot be corrected, the apnea test is not performed. In this case, the diagnosis is completed with an accessorial test such as a cerebral blood flow study.

$\mathrm{PaO}_{2}$ : arterial oxygen partial pressure, $\mathrm{ABGA}$ : arterial blood gas analysis, $\mathrm{SpO}_{2}$ : pulse oximetry saturation. 
$\mathrm{N}$-group, the ratio of normal $\mathrm{PaCO}_{2}$ was $15 / 28$ (53.6\%) the first time and 19/28 (67.9\%) the second time; there was a significant difference between the two groups.

During the apnea test, because $\mathrm{CO}_{2}$ in the blood cannot be released to alveoli, respiratory acidosis and hypercarbia begin to progress. Accumulated $\mathrm{CO}_{2}$ and acidosis caused by increased $\mathrm{CO}_{2}$ lead directly to reductions in peripheral arteriolar resistance, cardiac contractile force, and the rate of conduction. In the sympathetic system, however, the opposite action occurs. Aortic and carotid chemoreceptors stimulated by hypercarbia indirectly increase the activation of sympathetic tone, which improves cardiac contractile force and vascular resistance. Therefore, overall hemodynamic changes are impacted by a combination of direct and indirect effects. Because excessive acidosis reduces the sympathetic response of the human body, so the effect of cardiovascular depression by acidosis and hypercarbia exceed the effect of cardiovascular stimulation by sympathetic response $[24,25]$. Therefore, in delayed apnea status during apnea testing, hemodynamic instability progresses, and critical complications such as severe hypotension, arrhythmia, and cardiac arrest are likely to occur [3-5]. For that reason, it is necessary to minimize the apnea test period as well as maintain a normal range of $\mathrm{PaCO}_{2}$. The rate at which $\mathrm{PaCO}_{2}$ increases in the apnea test is non-linear; if $\mathrm{PaCO}_{2}$ is in the range of about 40$60 \mathrm{mmHg}$, the rate of increase is $4-6 \mathrm{mmHg} / \mathrm{min}$, and if $\mathrm{PaCO}_{2}$ is in a higher range, the rate of increase is $2 \mathrm{mmHg} / \mathrm{min}$. In addition, recent studies show variation in the rate of $\mathrm{PaCO}_{2}$ increase. Vivien et al. [18] reported $2.8 \pm 0.9 \mathrm{mmHg} / \mathrm{min}$, Edward et al. [26] reported $3.7 \pm 2.3 \mathrm{mmHg} / \mathrm{min}$, and Bruce [27] reported $1.7 \mathrm{mmHg} / \mathrm{min}$. This variation is caused by passive $\mathrm{CO}_{2}$ washout, atelectasis, cardiac-induced ventilations, and other unknown factors [26]. Thus, even though we know the value of $\mathrm{PaCO}_{2}$ before the apnea test, it is difficult to predict the time to target $\mathrm{PaCO}_{2}(50 \mathrm{mmHg})$ after the start of the test. We assume, however, that maintaining a normal $\mathrm{PaCO}_{2}$ level with $\mathrm{ETCO}_{2}$ monitoring can reduce the duration of the apnea test compared to no monitoring of $\mathrm{ETCO}_{2}$. As we expected, in the C-group, the time to target $\mathrm{PaCO}_{2}$ and total apnea test period were reduced significantly compared to the N-group (Table 2).

We also expected to reduce hemodynamic changes and hypoxemia by reducing the total test time in the C-group. To confirm the reduction of hemodynamic changes, we recorded SBPs at the beginning and end of the test and checked the differences. Differences in SBP during the test (SBP at the end of AT - SBP at the beginning of AT) were found in the N-group $\left(1^{\text {st }}\right.$ AT: $-9.0 \pm 26.2 \mathrm{mmHg}, 2^{\text {nd }}$ AT: $-12.4 \pm 26.8 \mathrm{mmHg}$ ) and in the C-group ( $1^{\text {st }}$ AT: $9.1 \pm 27.7 \mathrm{mmHg}, 2^{\text {nd }}$ AT: $\left.5.3 \pm 31.2 \mathrm{mmHg}\right)$. In the C-group, SBP increased at the end of the test compared to the beginning both times the test was administered. Although the usage of inotropics differed for each patient, the protocol regarding their use was the same-to maintain SBP at more than $100 \mathrm{mmHg}$. Thus, we think the reason that SBP increased only in the C-group is that the reduction in the total apnea test period and the prevention of excessive acidosis contributed to hemodynamic stability. As mentioned before, it is known that in the initial stage of the apnea test, a temporary increase in BP can appear due to sympathetic stimulation, and a decrease in BP appears as acidosis progresses. To confirm a temporary increase in $\mathrm{BP}$ in the initial stage of the apnea test, it would be necessary to look for a change in BP as time progressed in both groups, but we could not find the records of real-time BP during apnea testing. Thus, we instead compared the incidence of SBP under 100 $\mathrm{mmHg}$ with both groups according to whether $\mathrm{PaCO}_{2}$ was within the normal range. The incidence of SBP under $100 \mathrm{mmHg}$ during the first test (normal $\mathrm{PaCO}_{2}$ group 8/44 [18\%], abnormal $\mathrm{PaCO}_{2}$ group 2/13 [15\%]) and second test (normal $\mathrm{PaCO}_{2}$ group 6/48 [12\%], abnormal $\mathrm{PaCO}_{2}$ group 3/9 [33\%]) was identified. In short, we found no difference in the incidence of SBP under $100 \mathrm{mmHg}$ between the normal and abnormal $\mathrm{PaCO}_{2}$ groups. The ratio of $\mathrm{SpO}_{2}$ under $90 \%$ during the apnea test for the first test (N-group 3/28 [10.7\%], C-group 1/29 [3.4\%]) and second test (N-group 4/28 [14.3\%], C-group 4/29 [13.8\%]) was also determined. Although the $\mathrm{N}$-group had a higher incidence than the C-group, there was no significant difference.

The ultimate goal of this study was to minimize damage to organs planned for transplantation and thus contribute to successful organ transplantation. We thus examined whether available organs and final donated organs were different between the N-group and C-group. The discrepancy between available organs and final donated organs was 8/28 (29\%) in the N-group and $12 / 29(41 \%)$ in the C-group, not a significant difference ( $\mathrm{P}$ value 0.31 ). However, we could not evaluate the relationship between the hemodynamic stability of donors and the success rate of organ transplantation because of a number of variables we could not identify, including problems related to organ extraction from donors, status change of recipients, donor-recipient mismatch, and problems related to organ transportation. Such issues are left to further studies.

There are some limitations to the present study. First, because this is a small group study that examined only 57 patients, it is possible that a larger sample might yield statistically significant results that did not achieve significance in this study. Second, because we had trouble collecting data due to the nature of retrospective research, we could not confirm some variables we hoped to identify, such as hemodynamic changes during the apnea test and the rate of successful transplantation. Third, as the examinations were performed by various physicians, it is possible that different methods were used to diagnose brain death. The results showed differences in the interval from the beginning of the test to first blood sampling, and, if first $\mathrm{PaCO}_{2}$ 
did not reach target value, the interval from first blood sampling to second blood sampling. Thus, comparing the individual durations of the apnea tests is difficult. However, it remains the case that there were statistically significant differences between the groups. Fourth, because this investigation covered more than 3 years, differences in the skills of the examiner could occur with the passage of time.

In summary, we maintained $\mathrm{PaCO}_{2}$ within a normal range when monitoring $\mathrm{ETCO}_{2}$ before apnea testing. In addition, because maintaining $\mathrm{PaCO}_{2}$ within a normal range reduced the time to reach target $\mathrm{PaCO}_{2}$ from the beginning of the test, the total apnea test period was reduced. Based on the reduction of the test period, we confirmed that SBP increased more at the end of the test than the beginning in the group in which capnography was applied. Thus, when applying capnography in brain dead patients, we confirmed that the duration of the apnea test is reduced and hemodynamic stability is increased. In conclusion, $\mathrm{ETCO}_{2}$ monitoring with capnography is helpful when performing the apnea test.

\section{References}

1. Wijdicks EF. The diagnosis of brain death. N Engl J Med 2001; 344: 1215-21.

2. Scripko PD, Greer DM. An update on brain death criteria: a simple algorithm with complex questions. Neurologist 2011; 17: 237-40.

3. Saposnik G, Rizzo G, Vega A, Sabbatiello R, Deluca JL. Problems associated with the apnea test in the diagnosis of brain death. Neurol India 2004; 52: 342-5.

4. Wu XL, Fang Q, Li L, Qiu YQ, Luo BY. Complications associated with the apnea test in the determination of the brain death. Chin Med J (Engl) 2008; 121: 1169-72.

5. Wijdicks EF, Rabinstein AA, Manno EM, Atkinson JD. Pronouncing brain death: Contemporary practice and safety of the apnea test. Neurology 2008; 71: 1240-4.

6. Vivien B, Marmion F, Roche S, Devilliers C, Langeron O, Coriat P, et al. An evaluation of transcutaneous carbon dioxide partial pressure monitoring during apnea testing in brain-dead patients. Anesthesiology 2006; 104: 701-7.

7. Wooda KE, Coursin DB. Intensivists and organ donor management. Curr Opin Anaesthesiol 2007; 20: 97-9.

8. Casati A, Salvo I, Torri G, Calderini E. Arterial to end-tidal carbon dioxide gradient and physiological dead space monitoring during general anaesthesia: effects of patients' position. Minerva Anestesiol 1997; 63: 177-82.

9. Eskaros SM, Papadakos PJ, Lachmann B. Respiratory monitoring. In: Miller's anesthesia. 7th ed. Edited by Miller RD: Philadelphia, Churchill Livingstone. 2010, pp 1411-41.

10. Butterworth JF, Mackey DC, Wasnick JD. Noncardiovascular monitoring. In: Morgan \& Mikhail's clinical anesthesiology. 5th ed. Edited by Morgan GE, Mikhail MS: New York, McGraw-Hill. 2013, pp 125-7.

11. McSwain SD, Hamel DS, Smith PB, Gentile MA, Srinivasan S, Meliones JN, et al. End-tidal and arterial carbon dioxide measurements correlate across all levels of physiologic dead space. Respir Care 2010; 55: 288-93.

12. Ministry of health and wellfare. Enforcement decree of act on organ transplantation. Ministry of government legislation. Seoul, 2012.

13. Korea centers for disease control and prevention. Annual report of the transplant. Korean network for organ sharing. Seoul, Korean Network for Organ Sharing. 2012. Available from http://www.konos.go.kr/konosis.

14. Lang CJ, Heckmann JG. Apnea testing for the diagnosis of brain death. Acta Neurol Scand 2005; 112: 358-69.

15. Practice parameters for determining brain death in adults (summary statement). The Quality Standards Subcommittee of the American Academy of Neurology. Neurology 1995; 45: 1012-4.

16. Sullivan KJ, Kissoon N, Goodwin SR. End-tidal carbon dioxide monitoring in pediatric emergencies. Pediatr Emerg Care 2005; 21 : 327-32.

17. Blanch L, Romero PV, Lucangelo U. Volumetric capnography in the mechanically ventilated patient. Minerva Anestesiol 2006; $72: 577-85$.

18. Vivien B, Amour J, Nicolas-Robin A, Vesque M, Langeron O, Coriat P, et al. An evaluation of capnography monitoring during the apnoea test in brain-dead patients. Eur J Anaesthesiol 2007; 24: 868-75.

19. Hoffman RA, Krieger BP, Kramer MR, Segel S, Bizousky F, Gazeroglu H, et al. End-tidal carbon dioxide in critically ill patients during changes in mechanical ventilation. Am Rev Respir Dis 1989; 140: 1265-8.

20. Ward KR, Yealy DM. End-tidal carbon dioxide monitoring in emergency medicine, Part 1: Basic principles. Acad Emerg Med 1998; 5: 62836.

21. Kerr ME, Zempsky J, Sereika S, Orndoff P, Rudy EB. Relationship between arterial carbon dioxide and end-tidal carbon dioxide in mechanically ventilated adults with severe head trauma. Crit Care Med 1996; 24: 785-90.

22. Sharpe MD, Young GB, Harris C. The apnea test for brain death determination: an alternative approach. Neurocrit Care 2004; 1: 363-6.

23. Klingstedt C, Hedenstierna G, Baehrendtz S, Lundqvist H, Strandberg A, Tokics L, et al. Ventilation-perfusion relationships and atelectasis formation in the supine and lateral positions during conventional mechanical and differential ventilation. Acta Anaesthesiol Scand 1990; 34: 421-9.

24. Price HL. Effects of carbon dioxide on the cardiovascular system. Anesthesiology 1960; 21: 652-63. 
25. Goudreau JL, Wijdicks EF, Emery SF. Complications during apnea testing in the determination of brain death: predisposing factors. Neurology 2000; 55: 1045-8.

26. Benzel EC, Gross CD, Hadden TA, Kesterson L, Landreneau MD. The apnea test for the determination of brain death. J Neurosurg 1989; 71 : 191-4.

27. Bruce DL. Blood gas values change slowly in apneic organ donors. Anesthesiology 1986; 65: 128. 